\title{
FATORES ASSOCIADOS AO EXCESSO DE PESO DE ESCOLARES DA CIDADE DE PELOTAS-RS
}

\section{Factors associated with overweight of schoolers in Pelotas-RS}

Carla Ribeiro Ciochetto 1 , Fernanda de Oliveira Meller², Anelise Medeiros Souto', Maria de Fátima Alves Vieira ${ }^{1}$

${ }^{1}$ Faculdade de Nutrição, Universidade Federal de Pelotas, Brasil.

2 Programa de Pós-graduação em Saúde Coletiva, Universidade do Extremo Sul Catarinense, Brasil.

\section{Endereço para correspondência:}

Fernanda de Oliveira Meller

Av. Universitária, 1105, Criciúma - SC, CEP: 88806-000

Telefone: (48) 34312609

Email: fernandameller@unesc.net 


\title{
Resumo
}

O objetivo do estudo foi avaliar os fatores associados ao excesso de peso de escolares do ensino fundamental de escolas municipais da cidade de Pelotas/RS. Foi realizado um estudo transversal com 343 escolares com faixa etária entre 5 a 15 anos, do $1^{\circ}$ ao $4^{\circ}$ ano da rede pública municipal do ensino fundamental da cidade de Pelotas/RS. Para obtenção das variáveis demográficas, socioeconômicas e alimentares foi aplicado um questionário às mães ou responsáveis pelo escolar, em visita domiciliar. O estado nutricional foi avaliado através do índice de massa corporal por idade, em escore-z, e os escolares foram classificados em "sem excesso de peso" e "com excesso de peso". Todas as análises foram estratificadas por sexo. A prevalência de excesso de peso encontrada foi de $43,7 \%$ (46,7\% no sexo masculino e 40,3\% no sexo feminino). As variáveis escolaridade materna e nível socioeconômico apresentaram associação com o excesso de peso dos escolares na amostra total e em ambos os sexos. Além disso, as maiores prevalências de excesso de peso foram evidenciadas nos escolares que não realizavam o café da manhã. Políticas de promoção de saúde e de alimentação saudável são necessárias, a fim de prevenir o aumento da prevalência de excesso de peso entre os escolares.

Palavras-chave: Excesso de Peso; Fatores Associados; Escolares; Estudo Transversal.

\begin{abstract}
The objective of this study was to evaluate factors associated with overweight in primary public schools of the city of Pelotas, RS, Brazil. Was performed a crosssectional study with 343 schoolchildren aged between 5 to 15 years, from 1st to 4 th year of municipal public elementary schools in the city. To obtain the demographic, socioeconomic and dietary variables, a questionnaire was administered to mothers or guardians in a home visit. The nutritional status was assessed by body mass index for age in z-score, and the students were classified as "normal weight" and "overweight". All analyzes were stratified by sex. The prevalence of overweight found was $43.7 \%$ (46.7\% for males and $40.3 \%$ for females). The variables "maternal education" and "socioeconomic status" were associated with overweight students for the total sample and both sexes. Moreover, the highest prevalence of overweight was found among
\end{abstract}




\section{Atenção à Saúde}

students who did not eat breakfast. Health promotion and healthy eating policies are needed to prevent the increasing prevalence of overweight among students.

Keywords: Overweight; Risk Factors; School; Cross-Sectional Study.

\section{INTRODUÇÃO}

O processo de transição nutricional, ocorrido nas últimas décadas, caracterizou-se pela redução na prevalência dos déficits nutricionais e aumento da ocorrência de sobrepeso e obesidade ${ }^{1}$. O sedentarismo e as mudanças no estilo de vida e nos hábitos alimentares têm contribuído para o aumento da prevalência de excesso de peso $^{2,3}$, podendo levar a problemas de autoestima e de relacionamentos interpessoais, além de outras complicações ${ }^{4}$.

A alimentação adequada em quantidade e qualidade é essencial para que se obtenha a energia e os nutrientes necessários para o bom funcionamento do organismo, além de prevenir os distúrbios nutricionais ${ }^{5}$. Quando os hábitos alimentares não contemplam uma alimentação saudável, pode haver consumo alimentar insuficiente, gerando deficiências nutricionais ou consumo alimentar excessivo, predispondo à obesidade ${ }^{6,7}$.

Inúmeros fatores interferem na alimentação dos indivíduos, muitos deles tendo sua origem na infância e perpetuando-se ao longo da vida. Neste contexto, o ambiente escolar possui papel fundamental na formação dos hábitos alimentares, visto que grande parte de crianças e adolescentes permanecem pelo menos um turno por dia na escola, tornando-a um espaço privilegiado para o desenvolvimento de estratégias de promoção de hábitos saudáveis ${ }^{8}$.

Desta forma, o objetivo do presente trabalho foi avaliar os fatores associados ao excesso de peso de escolares do $1^{\circ}$ ao $4^{\circ}$ ano do ensino fundamental de escolas municipais da cidade de Pelotas/RS.

\section{METODOLOGIA}


O presente trabalho é parte de um estudo de intervenção que ocorreu no período de março a junho do ano de 2011, o qual avaliou o estado nutricional e os hábitos alimentares de escolares do $1^{\circ}$ ao $4^{\circ}$ ano do ensino fundamental da rede pública municipal da cidade de Pelotas, Rio Grande do Sul ${ }^{9}$.

O município de Pelotas possui 40 escolas com Ensino Fundamental na zona urbana da cidade. As escolas foram listadas em ordem alfabética e numeradas. Em seguida, seis foram sorteadas e, após a primeira seleção, foi realizado um segundo sorteio para selecionar uma turma por escola de $1^{\circ}, 2^{\circ}, 3^{\circ}$ ou $4^{\circ}$ ano do Ensino Fundamental.

Todos os escolares matriculados nas turmas sorteadas foram considerados elegíveis para o estudo. Foram excluídas as instituições de ensino que apresentaram turmas multisseriadas e que já participavam de projetos da Faculdade de Nutrição da Universidade Federal de Pelotas. Ao final do estudo, a amostra foi de 411 escolares.

As variáveis demográficas, socioeconômicas e alimentares foram coletadas através de um questionário aplicado à mãe ou ao responsável pelo escolar, em visita domiciliar. Todos os dados foram coletados por entrevistadoras previamente treinadas.

As informações demográficas coletadas foram sexo e idade, sendo que os alunos foram classificados em crianças ( 5 a 9 anos) e adolescentes (10 anos ou mais). Em relação às variáveis socioeconômicas, a escolaridade materna foi coletada em anos completos de estudo ( 0 a $4 ; 5$ a $8 ; 9$ a 11; 12 anos ou mais), e o nível socioeconômico foi coletado segundo as categorias utilizadas pela Associação Brasileira de Empresas de Pesquisas (ABEP) ${ }^{10}$. Devido ao reduzido número de indivíduos pertencentes às classes $A$ e $E$, a variável foi recategorizada em $A / B, C$ e D/E.

O número de refeições e a realização do café da manhã foram avaliados através da seguinte pergunta: "Quais as refeições que o estudante faz durante o dia?".

As medidas de peso e altura dos escolares foram aferidas no ambiente escolar, seguindo critérios de Lohman et al. ${ }^{11}$. Para obtenção do peso, utilizou-se balança solar portátil (Modelo 1631; TANITA®; Tóquio, Japão) com capacidade máxima de $150 \mathrm{~kg}$ e precisão de $0,2 \mathrm{~kg}$. Para aferição da altura, foi utilizada fita métrica inextensível de $150 \mathrm{~cm}$ de comprimento, afixada de forma invertida com fitas adesivas, em parede plana e sem rodapé, estando posicionada a $50 \mathrm{~cm}$ do chão. $O$ estado 


\section{Artigo Original}

\section{Atenção à Saúde}

nutricional foi avaliado através do Índice de Massa Corporal (IMC) por idade, em escore-z, obtido através do programa Anthro-Plus ${ }^{12}$. Os escolares foram classificados nas seguintes categorias: magreza $(<-2$ escore-z); eutrofia (entre $\geq-2$ e $\leq+1$ escore$z$ ); sobrepeso (entre $>+1$ e $\leq+2$ escore-z); obesidade (entre $>+2$ e $\leq+3$ escore-z) e obesidade grave $(>+3 \text { escore- } z)^{13}$. Para fins de análise estatística, o estado nutricional foi analisado de forma dicotômica, considerando "com excesso de peso" aqueles que apresentaram valores de IMC por idade $>+1$ escore- $z$ e "sem excesso de peso" aqueles com escore- $z \leq+1$.

O controle de qualidade dos dados foi feito pelo coordenador do trabalho de campo, através de entrevista telefônica com amostra aleatória de 10\% dos entrevistados, através da aplicação de algumas perguntas do questionário original. Os dados foram duplamente digitados no programa Epi Info 6.0 (Centers for Disease Controland Prevention, Atlanta, Estados Unidos) e analisados através do programa Stata versão 11.0. Para a análise estatística foi utilizado o teste de Qui-quadrado para heterogeneidade ou tendência linear $(p<0,05)$. Todas as análises foram estratificadas por sexo.

O estudo foi aprovado pelo Comitê de Ética em Pesquisa da Faculdade de Medicina da Universidade Federal de Pelotas, protocolo № 11/11. Além disso, obtevese autorização da Secretaria Municipal de Educação do Município e dos pais ou responsáveis pelos escolares, através de assinatura do Termo de Consentimento Livre e Esclarecido.

\section{RESULTADOS}

Dentre os 411 alunos considerados elegíveis para o estudo, houve $13,4 \%$ de perdas e recusas, totalizando 356 crianças e adolescentes avaliados. Com relação às perdas, $67 \%$ eram do gênero masculino e a metade ocorreu em uma única escola em razão da sua política de fornecimento de endereços dos escolares, o que impediu o acesso aos pais ou responsáveis para realização da entrevista. Para a grande maioria dos alunos foram obtidas as medidas de peso e de altura, totalizando 343 escolares estudados $(96,3 \%)$.

A Tabela 1 apresenta as características demográficas, socioeconômicas, alimentares e antropométrica dos estudantes. Observa-se que a maioria dos 
Artigo Original

Atenção à Saúde

escolares era do sexo masculino $(53,6 \%)$ e criança (80,5\%). Aproximadamente $20 \%$ das mães apresentavam até 4 anos de estudo e 1/4 dos escolares pertencia a famílias de classe A/B. Quanto às variáveis alimentares, dois terços dos estudantes fazia 5 a 6 refeições diárias $(66,2 \%)$ e a maioria realizava o café da manhã $(78,4 \%)$. Em relação ao estado nutricional dos alunos, quase metade deles $(43,7 \%)$ apresentava excesso de peso (46,7\% no sexo masculino e $40,3 \%$ no sexo feminino).

Tabela 1. Características demográficas, socioeconômicas, de comportamento alimentar e antropométrica dos escolares. Estudo com escolares de $1^{\circ}$ ao $4^{\circ}$ ano do ensino fundamental ( $n=343)$. Pelotas, Brasil, 2011.

\begin{tabular}{lcc}
\hline Variáveis & $\mathbf{n}$ & $\%$ \\
\hline Sexo & & \\
$\quad$ Masculino & 184 & 53,6 \\
$\quad$ Feminino & 159 & 46,4 \\
Faixa etária & & \\
$\quad$ Criança & 276 & 80,5 \\
$\quad$ Adolescente & 67 & 19,5 \\
Escolaridade materna (anos completos) & & \\
O a 4 & 63 & 18,4 \\
5 a 8 & 131 & 38,2 \\
9 a 11 & 131 & 38,2 \\
12 ou mais & 18 & 5,2 \\
Nível socioeconômico & & \\
A/B & 81 & 23,6 \\
C & 217 & 63,3 \\
D/E & 45 & 13,1 \\
Número de refeições & & \\
$\quad$ Até 4 & 116 & 33,8 \\
5 & 142 & 41,4 \\
6 & 85 & 24,8 \\
Realiza o café da manhã & & \\
Sim & 269 & 78,4 \\
Não & 74 & 21,6 \\
Excesso de peso & & \\
Sim & 150 & 43,7 \\
Não & 193 & 56,3 \\
\hline
\end{tabular}

Não houve observações desconhecidas para nenhuma das variáveis estudadas. 
$\mathrm{Na}$ Tabela 2, estão apresentadas as prevalências de excesso de peso nos escolares segundo as variáveis independentes, na amostra total e estratificada por sexo. As variáveis escolaridade materna, nível socioeconômico e realização do café da manhã mostraram-se associadas ao excesso de peso na amostra total e em ambos os sexos. A associação com a escolaridade materna tanto na amostra total $(p=0,002)$ quanto no sexo masculino $(p=0,008)$ apresentaram tendência linear direta, ou seja, quanto maior a escolaridade materna, maiores as prevalências de excesso de peso nos escolares. Em relação à associação do excesso de peso com o nível socioeconômico, também foi observada tendência linear direta na amostra total $(p<0,001)$. Além disso, os escolares que não realizavam café da manhã apresentaram maiores prevalências de excesso de peso $(p=0,001)$. As variáveis faixa etária e número de refeições não se mostraram associadas ao excesso de peso dos escolares.

Tabela 2. Prevalência de excesso de peso nos escolares segundo variáveis independentes, na amostra total e estratificada por sexo. Estudo com escolares de $1^{\circ}$ ao $4^{\circ}$ ano do ensino fundamental ( $\left.\mathrm{N}=343\right)$. Pelotas, Brasil, 2011.

\begin{tabular}{|c|c|c|c|c|c|c|c|c|c|}
\hline \multirow[t]{2}{*}{ Variáveis } & \multicolumn{3}{|c|}{ Todos } & \multicolumn{3}{|c|}{ Masculino } & \multicolumn{3}{|c|}{ Feminino } \\
\hline & $\mathbf{N}$ & $\%$ & Valor $\mathbf{p}$ & $\mathbf{N}$ & $\%$ & Valor $\mathbf{p}$ & $\mathbf{N}$ & $\%$ & Valor $p$ \\
\hline Faixa etária & & & $0,218^{a}$ & & & $0,913^{a}$ & & & $0,075^{a}$ \\
\hline Criança & 116 & 42,0 & & 67 & 46,5 & & 49 & 37,1 & \\
\hline Adolescente & 34 & 50,7 & & 19 & 47,5 & & 15 & 55,6 & \\
\hline $\begin{array}{l}\text { Escolaridade materna (anos } \\
\text { completos) }\end{array}$ & & & $0,002^{b}$ & & & $0,008^{b}$ & & & $0,017^{\mathrm{a}}$ \\
\hline 0 a 4 & 21 & 33,3 & & 12 & 32,4 & & 9 & 34,6 & \\
\hline 5 a 8 & 49 & 37,4 & & 30 & 44,8 & & 19 & 29,7 & \\
\hline 9 a 11 & 70 & 53,4 & & 36 & 51,4 & & 34 & 55,7 & \\
\hline 12 ou mais & 10 & 55,7 & & 8 & 80,0 & & 2 & 25,0 & \\
\hline Nível socioeconômico & & & $<0,001^{b}$ & & & $<0,001^{b}$ & & & $0,039^{a}$ \\
\hline $\mathrm{A} / \mathrm{B}$ & 52 & 64,2 & & 2 & 8,7 & & 8 & 36,4 & \\
\hline C & 88 & 40,5 & & 54 & 45,8 & & 34 & 34,4 & \\
\hline $\mathrm{D} / \mathrm{E}$ & 10 & 22,2 & & 30 & 69,8 & & 22 & 57,9 & \\
\hline Número de refeições & & & $0,541^{a}$ & & & $0,738^{a}$ & & & $0,543^{a}$ \\
\hline Até 4 & 54 & 46,6 & & 30 & 47,6 & & 24 & 45,3 & \\
\hline 5 & 63 & 44,4 & & 34 & 49,3 & & 29 & 39,7 & \\
\hline 6 & 33 & 38,8 & & 22 & 42,3 & & 11 & 33,3 & \\
\hline Realiza o café da manhã & & & $0,001^{\mathrm{a}}$ & & & $0,025^{a}$ & & & $0,014^{a}$ \\
\hline Não & 45 & 60,8 & & 26 & 61,9 & & 19 & 59,4 & \\
\hline $\operatorname{Sim}$ & 105 & 39,0 & & 60 & 42,2 & & 45 & 35,4 & \\
\hline
\end{tabular}

a teste qui-quadrado para heterogeneidade

${ }^{\mathrm{b}}$ teste qui-quadrado para tendência linear 
Destaca-se como ponto positivo do trabalho o fato de ser uma amostra representativa dos estudantes das escolas municipais de Pelotas, Rio Grande do Sul.

Um importante e preocupante achado do estudo foi a alta prevalência de excesso de peso entre os escolares avaliados (43,7\%), sendo $46,7 \%$ entre os meninos e $40,3 \%$ entre as meninas. Tal prevalência foi maior do que a encontrada em escolares brasileiros, os quais apresentaram $23,2 \%$ de excesso de peso (16\% apresentam sobrepeso e $7,2 \%$ obesidade) ${ }^{14}$.

Segundo os dados da Pesquisa de Orçamentos Familiares de 2008 e 2009, uma em cada três crianças brasileiras de 5 a 9 anos de idade está acima do peso. Em relação aos adolescentes, o excesso de peso foi diagnosticado em cerca de $20 \%$ deles ( $21,7 \%$ no sexo masculino e $19,4 \%$ no feminino), excedendo em seis vezes a frequência do déficit de peso. Em relação à obesidade, a prevalência foi de 5,9\% e $4 \%$ para os adolescentes do sexo masculino e feminino, respectivamente ${ }^{15}$. No presente estudo também foi avaliado o excesso de peso por faixa etária, encontrando $42,0 \%$ nas crianças e 50,7\% nos adolescentes, sendo utilizado os mesmos critérios de avaliação da Pesquisa de Orçamentos Familiares de 2008 e 2009.

Estudo realizado, em 2004, na cidade de Pelotas-RS encontrou cerca de 30\% de prevalência de excesso de peso em escolares do ensino fundamental ${ }^{16}$. Tal estudo avaliou escolas públicas e privadas, e encontrou menores prevalências de excesso de peso nas escolas municipais. No presente estudo foram avaliadas somente escolas municipais, e a prevalência supera os dados de 2004 em 16,5\%. Esta comparação deve ser feita com cautela, pois os estudos utilizaram diferentes métodos de avaliação nutricional. No estudo de Pelotas ${ }^{16}$ a avaliação nutricional foi feita segundo a proposta da International Obesity Task Force (IOTF) ${ }^{17}$, ao passo que no presente estudo foram utilizadas as recomendações da Organização Mundial de Saúde ${ }^{13}$.

Diferenças nas prevalências de excesso de peso entre os sexos têm sido observadas em estudos nacionais, ambos encontrando maiores prevalências no sexo masculino ${ }^{18,19}$. Por outro lado, segundo os dados da Pesquisa de Orçamentos Familiares de 2008 e 2009, o excesso de peso entre os sexos foi semelhante, com prevalências de $34,8 \%$ (masculino) e $32 \%$ (feminino) entre as crianças, e $21,7 \%$ (masculino) e 19,4\% (feminino) entre os adolescentes ${ }^{15}$. Outros estudos também não 


\section{Atenção à Saúde}

evidenciaram diferença nas prevalências de excesso de peso entre os sexos de crianças e adolescentes ${ }^{20,21,22,23}$, corroborando com os resultados do presente trabalho.

Outro resultado encontrado no presente estudo foi a associação direta entre escolaridade materna e excesso de peso dos escolares de ambos os sexos. Resultados semelhantes foram encontrados em estudo com escolas da rede pública, onde foi encontrada uma associação positiva entre a escolaridade materna e o excesso de peso, caracterizando o importante papel da mãe no processo de desenvolvimento dos filhos ${ }^{18}$. Em contrapartida, alguns autores não encontraram associação entre essas variáveis ${ }^{24,25}$.

Quanto ao nível socioeconômico, encontrou-se maior prevalência de excesso de peso nos escolares pertencentes às famílias de classes $A / B(64,2 \%)$, seguida pela classe C e D/E, com 40,5\% e 22,2\%, respectivamente. Dados semelhantes foram observados em outros estudos ${ }^{15,26,27}$. A Pesquisa de Orçamentos Familiares de 2008 e 2009 encontrou um aumento do excesso de peso naquelas crianças e adolescentes pertencentes a famílias de maior renda quando comparadas às de baixa e média renda ${ }^{15}$. Em contrapartida, há estudos que mostram que o excesso de peso vem crescendo nos países menos desenvolvidos e em populações com menor nível socioeconômico ${ }^{28,29}$. Tal fato pode ser atribuído ao baixo custo de alguns alimentos, que apresentam elevado teor de gordura, sal e açúcar, além da alta densidade energética e baixo valor nutritivo. Estes padrões de consumo alimentar, associado ao sedentarismo, resultam no sobrepeso e obesidade ${ }^{30}$.

Foi evidenciada maior prevalência de excesso de peso entre aqueles escolares que não realizavam o café da manhã. Estudo realizado com escolares também evidenciou resultado semelhante ${ }^{31}$. Omitir o desjejum é uma tentativa equivocada de reduzir calorias. $O$ consumo diário do café da manhã pode controlar o peso corporal devido ao menor consumo de gorduras na dieta em função do papel minimizador no consumo de lanches mais energéticos ${ }^{32}$.

No presente estudo não foi observada associação entre faixa etária e excesso de peso, corroborando com os dados apresentados por Oliveira e Fisberg ${ }^{33}$ e Farias e Silva ${ }^{34}$. Em contrapartida, Leal et al. ${ }^{35}$ observaram maior prevalência de excesso de peso entre os adolescentes do que entre as crianças. Sugere-se que tal fato esteja relacionado à maior autonomia dos adolescentes na escolha dos alimentos quando 
Artigo Original

Atenção à Saúde

comparados às crianças, cujas escolhas alimentares na maioria das vezes são feitas pelos pais ou responsáveis. Desta forma, os adolescentes apresentam maior consumo de alimentos de alto valor calórico e baixo valor nutricional. Uma possível explicação para o resultado encontrado no presente estudo pode ser o fato de que os adolescentes avaliados estavam na fase inicial da adolescência e talvez ainda não tenham apresentado esse comportamento.

Conclui-se que a prevalência de excesso de peso entre os estudantes foi maior do que as prevalências descritas em escolares brasileiros nos últimos anos e maiores do que as observadas em estudo local do ano de 2004. O excesso de peso entre os escolares mostrou-se associado à escolaridade materna, ao nível socioeconômico e à não realização do café da manhã. Diante desse quadro, fica evidente a necessidade da adoção de políticas de promoção de saúde e de alimentação saudável, tanto no âmbito familiar quanto escolar, como forma de prevenir o avanço nas prevalências de excesso de peso entre os escolares.

\section{CONTRIBUIÇÃO DOS AUTORES}

Fernanda de Oliveira Meller e Maria de Fátima Alves Vieira contribuíram com as análises e revisão do artigo. Carla Ribeiro Ciochetto e Anelise Medeiros Souto participaram de todas as etapas da produção do artigo.

\section{REFERÊNCIAS}

1. Monteiro CA, Conde WL. Tendência secular da desnutrição e da obesidade na infância na cidade de São Paulo (1974-1996). Rev Saude Publica. 2010;34:433-9.

2. Escrivão MAMS, Oliveira FLC, Taddei JAAC, Lopez FA. Obesidade exógena na infância e na adolescência. J Pediatr. 2000;76:305-10.

3. Pimenta APA, Palma A. Perfil epidemiológico da obesidade em crianças: relação entre televisão, atividade física e obesidade. Rev Bras Cien Mov. 2001;9:19-24.

4. Triches RM, Giugliani ERJ. Insatisfação corporal em escolares de dois municípios da região Sul do Brasil. Rev Nutr. 2007;20:119-28.

5. Monteiro CA, Mondini L. Mudanças no padrão de alimentação da população urbana brasileira (1962-1988). Rev Saude Publica 1994;28:433-39. 
6. Monteiro CA, Mondini L, Costa RBL. Mudanças na composição e adequação nutricional da dieta familiar nas áreas metropolitanas no Brasil (1988-1996). Rev Saude Publica. 2010;34:251-8.

7. Coutinho JG, Gentil PC, Toral N. Malnutrition and obesity in Brazil: dealing with the problem through a unified nutritional agenda. Cad Saude Publica. 2008;24 Suppl 2:332-40.

8. Brasil. Ministério da Saúde. Organização Pan-americana de saúde. Escolas promotoras da saúde: experiências do Brasil. Brasília; 2006.

9. Ciochetto CR, Orlandi SP, Vieira MFA. Consumo de frutas e vegetais em escolares da rede pública no Sul do Brasil. Arch Latinoamer Nutr. 2012;62:172-8.

10. Associação Brasileira de Empresas de Pesquisa. Critério de classificação econômica $\quad$ Brasil $\quad 2011 . \quad$ Disponível em: http://www.abep.org/novo/Content.aspx?ContentlD=302

11. Lohman T, Roche A, Martorell R. Anthropometric standardization reference manual. Champaign: Human Kinetics, 1998.

12. World Health Organization. Anthro Plus for personal computers manual: Software for assessing growth of the world's children and adolescents. Geneva: WHO; 2009.

13. World Health Organization. WHO Growth reference data for 5-19 years. Geneva: WHO; 2007.

14. Brasil. Instituto Brasileiro de Demografia e Estatística. Pesquisa Nacional de Saúde do Escolar (PeNSE) 2009. Disponível em: http://www.ibge.gov.br/home/estatistica/populacao/pense/pense.pdf

15. Brasil. Instituto Brasileiro de Demografia e Estatística. Pesquisa de orçamento familiar 2008-2009: antropometria e estado nutricional das crianças, adolescentes e adultos no Brasil. Rio de Janeiro; 2010.

16. Vieira MFA, Araújo CLP, Hallal PC, Madruga SM, Neutzling MB, Matijasevich A, et al. Estado nutricional de escolares de $1^{\underline{a}}$ a $4^{\underline{a}}$ séries do Ensino Fundamental das escolas urbanas da cidade de Pelotas, Rio Grande do Sul, Brasil. Cad Saude Publica. 2008;24:1667-74.

17. Cole TJ, Bellizzi MC, Flegal KM, Dietz WH. Establishing a standard definition for child overweight and obesity worldwide: international survey. BMJ. 2000;320:1240-3.

18. Fernandes RA, Codogno JS, Cardoso JR, Ronque VER, Freitas Júnior IF, Oliveira AR. Fatores associados ao excesso de peso entre adolescentes de diferentes redes de ensino do município de Presidente Prudente, São Paulo. Rev Bras Saude Mater Infant. 2009;9:443-9. 


\section{Atenção à Saúde}

19. Farias ES, Santos AP, Farias-Júnior JC, Ferreira CRT, Carvalho WRG, Gonçalves EM, et al. Excesso de peso e fatores associados em adolescentes. Rev Nutr. 2012;25:229-36.

20. Dutra CL, Araújo CL, Bertoldi AD. Prevalence of overweight in adolescents: a population-based study in a southern Brazilian city. Cad Saude Publica. 2006;22:15162.

21. Suñé FR, Dias-da-Costa JS, Olinto MTA, Pattussi MP. Prevalência e fatores associados para sobrepeso e obesidade em escolares de uma cidade no Sul do Brasil. Cad Saude Publica. 2007;23:1361-71.

22. Brasil LMP, FisbergM, MarranoHS. Excesso de peso de escolares em região do Nordeste Brasileiro: contraste entre as redes de ensino pública e privada. Rev Bras Saude Mater Infant. 2007;7:405-12.

23. Lunardi CC, Moreira CM, Santos DL. Colesterolemia, trigliceridemia e excesso de peso em escolares de Santa Maria, RS, Brasil. Rev Bras Med Esp. 2010;16:250-3.

24. Plachta-Danielzik S, Landsberg $B$, Johannsen $M$, Lange D, Müller MJ. Determinants of the prevalence and incidence of overweight in children and adolescents. Public Health Nutr. 2010;13:1870-81.

25. Meller FO, MadrugaSW, Araújo CLP. Fatores associados ao excesso de peso em crianças brasileiras: PNDS 2006. Rev Ciencia Saude Coletiva. 2014;19:943-55.

26. Guimarães ACA, Feijó I, Soares A, Fernandes S, Machado Z, Parcias SR. Excesso de peso e obesidade em escolares: associação com fatores biopsicológicos, socioeconômicos e comportamentais. Arq Bras Endocrinol Metab. 2012;56:142-48.

27. Rosaneli CF, Auler F, Manfrinato CB, Rosaneli CF, Sganzerla C, Bonatto MG, et al. Avaliação da prevalência e de determinantes nutricionais e sociais do excesso de peso em uma população de escolares: análise transversal em 5.037 crianças. Rev Assoc Med Bras. 2012;58:472-6.

28. Nunes MMA, Figueiroa JN, Alves JGB. Excesso de peso, atividade física e hábitos alimentares entre adolescentes de diferentes classes econômicas em Campina Grande (PB). Rev Assoc Med Bras. 2007;53:130-4.

29. Alves JGB, Siqueira PP, Figueiroa JN. Overweight and physical inactivity in children living in favelas in the metropolitan region of Recife, Brazil. J Pediatr. 2009;85:67-71.

30. World Health Organization. Obesidad y sobrepeso. Disponível em: http://www.who.int/mediacentre/factsheets/fs311/es/

31. Triches RM, Giugliani ERJ. Obesidade, práticas alimentares e conhecimentos de nutrição em escolares. Rev Saude Publica. 2005;39:541-7. 
Artigo Original

Atenção à Saúde

32. Nicklas TA, Baranowsky T, Cullen KW, Berenson G. Eating patterns, dietary quality and obesity. J Am Coll Nutr. 2001;20:599-608.

33. Oliveira CL, Fisberg M. Obesidade na infância e adolescência: uma verdadeira epidemia. Arq Bras Endocrinol Metab. 2003;47:107-8.

34. Farias Júnior JC, Silva KS. Sobrepeso/obesidade em adolescentes escolares da Cidade de João Pessoa - PB: prevalência e Associação com Fatores Demográficos e Socioeconômicos. Rev Bras Med Esp. 2008;14:104-8.

35. Leal VS, Lira PIC, Oliveira JS, Menezes RCE, Sequeira LAS, Arruda-Neto MA, et al. Excesso de peso em crianças e adolescentes no Estado de Pernambuco, Brasil: prevalência e determinantes. Cad Saude Publica. 2012;6:1175-82. 